\title{
Diversity and Distribution of Clavarioid Fungi in India, Three Fungi from Central India
}

\author{
R.K. Verma* and Vimal Pandro
}

Forest Pathology Discipline, Forest Protection Division, Tropical Forest Research Institute, Jabalpur - 482 021, Madhya Pradesh, India

*Corresponding author

\section{A B S T R A C T}

\section{Keywords}

Club fungi, Coral fungi,

Ectomycorrhizal,

Eduble fungi,

Lichen forming

fungi

Article Info

Accepted:

15 November 2018

Available Online:

10 December 2018
Total 108 Indian clavarioid fungi belonging to 17 genera were compiled from literature. These fungi are distributed in 13 states of India. Genus Ramaria represent the largest number of species (39) followed by Clavaria (13), Clavulinopsis (12), Clavulina (11) and Typhula (10). Genera like Aphelaria, Artomyces, Deflexula, Gloeocantharellus, Multiclavula and Scytinopogon are least diverse and represented by only one species. Clavarioid fungi were recorded from 131 places of 13 states in India and the maximum diversity of these fungi was shown by the state Uttarakhand (57) followed by Himachal Pradesh (25) and West Bengal (10). Three clavarioid fungi namely, Phaeoclavulina flaccida, Ramariopsis kunzei and $R$. subtilis were described from Madhya Pradesh and Maharashtra. Earlier these fungi were reported from Uttarakhand and Chhattisgarh.

\section{Introduction}

The clavarioid fungi are a group of fungi in the Basidiomycota typically having erect, simple or branched basidiocarps (fruit bodies) that are formed on the ground, on decaying vegetation, or on dead wood. They are colloquially called club fungi and coral fungi. Originally such fungi were referred to the genus Clavaria, although clavarioid species are not all closely related and are often studied as a group. Therefore, it is convenient to retain the informal (non-taxonomic) name of "clavarioid fungi" and this term is frequently used in research papers.

The clavarioid fungi are classified in 9 families namely, Aphelariaceae, Clavariaceae, Clavulinaceae, Clavariadelphaceae, Gomphaceae, Lachnocladiaceae, Lentariaceae, Pterulaceae, and Typhulaceae. At present 35 genera of clavarioid fungi are known out of them 16 genera (Aphelaria, Artomyces, Clavaria, Clavariadelphus, Clavulina, Clavulinopsis, Deflexula, Gloeocantharellus, Lachnocladium, Lentaria, Multiclavula, 
Phaeoclavulina, Ramaria, Ramariopsis, Scytinopogon and Typhula) are known from India. Most of the clavarioid fungi are saprotrophic in nature with terrestrial habit. These fungi grow on variety of substrates in nature for example deadwood, leaf litter or on mossy grassland, soil, etc. Some species, particularly in the genera Clavulina and Ramaria, are also reported as ectomycorrhizal. Some of clavarioid fungi are lichenized with algae (e.g. Ertzia, Lepidostroma, Multiclavula, Sulzbacheromyces).

Most clavarioid fungi have simple or branched fruit bodies that are erect (or pendant from wood in the genus Deflexula). The spores are born on the sides of the clubs or branches and the spore-bearing surface is typically smooth or ridged, occasionally has wart or weak spiny surface. Ramaria is the largest genus, which has species with branched fruit bodies and ochre to brownish spores. Clavaria delphus, producing large, club-shaped fruit bodies (Humpert et al., 2001). Clavaria in its modern sense is restricted to white-spored species, many simple, some branched. It is not clearly distinguished from two related genera, Clavulinopsis and Ramariopsis (Dentinger and McLaughlin, 2006).

The genus Typhula contains a number of small, sometimes minute species with simple fruit bodies. The major genera of clavarioid fungi are Clavaria, Clavulinopsis, Ramaria, Ramariopsis and Typhula. Some genera have fewer species for example Aphelaria, Artomyces, Clavicorona, Clavulina, Lachnocladium, Lentaria, Multiclavula, Scytinopogon, etc. Clavarioid fungi have a worldwide distribution, although some genera such as Aphelaria and Lachnocladium are principally tropical.

The present article reports 108 clavarioid fungi belonging to 17 genera and distributed in India. These fungi were recorded from 131 places in 13 states and the maximum diversity was shown by Uttarakhand (57) followed by Himachal Pradesh (25) and West Bengal (11). Phaeoclavulina flaccida, Ramariopsis kunzei and $R$. subtilis were described from Madhya Pradesh and Maharashtra.

\section{Materials and Methods}

Specimens of Clavarioid fungi were collected from Madhya Pradesh and Maharashtra during rainy seasons. A part of collected samples were preserved in $70 \%$ alcohol just after collection for microscopic study.

The fruit bodies of fungi were dried under the sun or in the wooden or cardboard box lighted with $100 \mathrm{~W}$ electric bulb. Microscopic slides were prepared by using stain, mountant, clearing and softening chemicals. Slides were observed under advanced research microscope (Leica, Germany) using 5x, 10x, 20x, 40x objectives and 10x and 15x eyepieces. Observations under phase contrast and dark field were also made whenever required.

Photomicrography was done with the help of a digital camera (make, Leica) attached to the advanced microscope. Identification of fungi have been done with the help of published literature, monographs, books, keys, etc. (Banerjee, 1947; Banerjee and Ganguly, 1945; Berkeley, 1852a b; 1856; Corner, 1966; Corner et al., 1956; 1957; 1958; Corner and Thind, 1961; Das et al., 2016; De, 1991; Dehariya et al., 2010; Hennings, 1901; Joseph and Manimohan, 1998; Khurana, 1980; Khurana and Thind, 1979; Methven, 1989; Parndekar, 1964; Rattan and Khurana, 1978; Sharda, 1984; Sharma and Munjal, 1977; Thind and Anand, 1956a b c; Thind and Raswan, 1958; Thind and Rattan, 1967; Thind and Sharda, 1982; 1984; 1986, 1987; Thind and Sukh Dev, 1956; 1957; 1957a b; Tiwari et al., 2013) and matter available on web site. 


\section{Results and Discussion}

\section{Taxonomic description}

Phaeoclavulina flaccida (Fr.) Giachini (Figures 1-4)

$=$ Ramaria flaccida (Fr.) Bourdot

Elavaria flaccida $\mathrm{Fr}$.

=Clavariella flaccida (Fr.) P. Karst.

Gregarious, rarely solitary, medium sized, rarely large sized, radial, slender, flaccid, trunk absent, sometimes present, profusely branched, fleshy, smooth, glabrous, yellowish brown, dirty-brown; or dirty yellowish brown, trunk when present, slender up to $1.7 \mathrm{~cm}$ and up to $3 \mathrm{~mm}$.broad, branching dichotomous, branches slender, unequal, in alternating planes, sometimes very small or ligulate. Often fused with one another, ligulate or adventitious branches present all over the fructification and become bushy, primary branches slender, only up to $2.5 \mathrm{~mm}$ broad. Ultimate branchlet thin, small, unequal pairs sometimes minute and ligulate, very minute or 3-20mm long, apices concolors acute, fertile, flesh lighter colored taste and smell imperticular. Numerous rhizomorphic mycellial thread given out from the base of fructification. Hyphae monomitic, 2-8 $\mu \mathrm{m}$ broad, hyaline, branched, thin walled, septate, septa at long intervals, non inflated, or sometimes slightly inflated, considerably swollen into sac like structures at places near the ends or at the septa, clamped, clamp prominent. Hymenium spread all over except the lighter colored base, compound with numerous embedded spores usually in clusters of four, 70-105 $\mu \mathrm{m}$ thick. Basidia 4-7 $\mu \mathrm{m}$ broad, clavate, sterigmata four, slightly incurved, 3-6 $\mu \mathrm{m}$ long. Basidiospore 4-7 x 3$4 \mu \mathrm{m}$, small ochraceous to brown, ellipsoid, papillate, profusely echinulate, wall dark, aguttate.

\section{Collection examined}

On living stem (butt region) of 12 years old Tectona grandis, TFRI, Jabalpur, 16/8/2007, Tropical Forest Research Institute, TF 822

Ramariopsis kunzei (Fr.) Corner (Figures 58)

$\equiv$ Clavaria kunzei Fr.

=Clavulina kunzei (Fr.) J. Schröt.

=Ramaria kunzei (Fr.) Quél.

=Clavulinopsis kunzei (Fr.) Jülich

This coral like mushroom has a stout, whitish base and densely packed, short branches with pink tips, elongated branches and hard fruiting body $2-6.5 \mathrm{~cm}$, long and $3-4.5 \mathrm{~cm}$ wide, stocky repeatedly short branched, densely packed, basal branches thick, smooth and whitish; terminal branches crowded, short, pinkish, tips cauliflower like, when young, mycelium are septate, $\quad 3.7-6.2 \mu \mathrm{m}$ wide. Spores are transparent, irregular shape, measuring 5-12 x 4.5-7.5 $\mu \mathrm{m}$.

\section{Collection examined}

On the ground under Acacia melonoxylon, Near Devi Point $\left(21^{0} 23{ }^{\prime} 43^{\prime}\right.$ 'N $77^{0} 19^{\prime} 47^{\prime}$ 'E, Elevation $1075.36 \quad$ M.) Chikhaldara, Maharashtra, 11/10/2018, specimen deposited in Mycology Herbarium, Tropical Forest Research Institute, TF- 4062

\section{Ramariopsis subtilis (Pers.) R.H. Petersen (Figures 9-12)}

\section{$\equiv$ Clavaria subtilis Pers.}

Fruit bodies up to $4 \mathrm{~cm}$ high and up to $2 \mathrm{~cm}$ broad, branched, branching dichotomously throughout. Stipe up to $2 \mathrm{~cm}$ long and up to $2 \mathrm{~mm}$ thick, equal or tapering slightly downwards, white - cream, not brittle, subiculum white, spreading up to $1.5 \mathrm{~mm}$ from 
stipe base, effuse. Branches up to $1.5 \mathrm{~mm}$ thick, ascending, bone white, terete, axile narrowly angled below, acutely angled to rounded in ultimate rank, offen decurrent by a depressed line, apices swollen, irregularly lobed or cusped to subturbinate up to $2 \mathrm{~mm}$ broad. Tramal hyphae 1.5-5.5 $\mu \mathrm{m}$ diam, parallel, clamped, tightly packed, hyaline, thin walled. Basidia clavate, clamped, often sinuate, contents divergent, subcoronate, thick walled, minutely granular or with several refringent guttules, sterigmata four, deep orange ochre under phase contrast, $60-90 \mathrm{x}$ 7-9 $\mu \mathrm{m}$. Basidiospores globose to very broadly ellipsoid, hyaline, thin to somewhat thick walled (wall up to $0.3 \mu \mathrm{m}$ ), contents with a single large refringent guttule, spicules prominent, $2 \mu \mathrm{m}$ or more longer, 5.5-7.5 $\mathrm{x}$ 5.5-7.0 $\mu \mathrm{m}$.

\section{Collection examined}

On humus soil near tree stems and hedge, TFRI campus, Jabalpur, MP, 24/7/2013,
Tropical Forest Research Institute TF- 3448 and under Pongamia pinnata, 28/09/2017.

Phaeoclavulina flaccida, Ramariopsis kunzei and $R$. subtilis were described from Madhya Pradesh and Maharashtra. From India 108 clavarioid fungi spread over 17 genera were recorded. These fungi were distributed in 13 states. Clavarioid fungi from were recorded from 130 places of 13 states and the maximum diversity of occurrence these fungi was shown by the Uttarakhand state (57) followed by Himachal Pradesh (25) and West Bengal (10). On the contrary from states like Andhra Pradesh, Chhattisgarh and Uttar Pradesh only one species were reported. The largest genus recorded was Ramaria comprising 39 species followed by Clavaria 13 species, Clavulinopsis 12 species, Clavulina 11 species and Typhula 10 species, where as genera like Aphelaria, Artomyces, Deflexula, Gloeocantharellus, Multiclavula and Scytinopogon are least diverse and represented by only one species (Table 1,2).

Table.1 Clavarioid fungi reported from India

\begin{tabular}{|c|c|c|c|c|}
\hline S.No. & Name of fungus & Host/ substrate & Locality & Reference \\
\hline 1. & $\begin{array}{l}\text { Aphelaria tuberosa }(\mathrm{Grev} .) \\
\text { Corner } \equiv \text { Merisma tuberosum } \\
\text { Grev. }\end{array}$ & $\begin{array}{l}\text { on soil amid } \\
\text { mosses }\end{array}$ & $\begin{array}{l}\text { Mussoorie, } \\
\text { Uttarakhand }\end{array}$ & $\begin{array}{l}\text { Thind and Sukh Dev } \\
\text { (1956) }\end{array}$ \\
\hline 2. & $\begin{array}{l}\text { Artomyces pyxidatus } \text { (Pers.) } \\
\text { Jülich =Clavaria pyxidata } \\
\text { Pers. =Clavicorona pyxidata } \\
\text { (Pers.) Doty }\end{array}$ & on wood & $\begin{array}{l}\text { Mussoorie, } \\
\text { Uttarakhand }\end{array}$ & $\begin{array}{l}\text { Butler and Bisby } \\
\text { (1931) }\end{array}$ \\
\hline 3. & Clavaria acuta Sowerby & on soil & $\begin{array}{l}\text { Mussoorie, } \\
\text { Uttarakhand }\end{array}$ & $\begin{array}{l}\text { Thind and Sukh Dev } \\
\text { (1956) }\end{array}$ \\
\hline 4. & $\begin{array}{l}\text { Clavaria amoenoides Corner, } \\
\text { K.S. Thind \& Anand }\end{array}$ & $\begin{array}{l}\text { on soil in pine oak } \\
\text { forest }\end{array}$ & $\begin{array}{l}\text { Mussoorie, } \\
\text { Uttarakhand }\end{array}$ & Corner et al., (1956) \\
\hline 5. & $\begin{array}{l}\text { Clavaria angulispora Pat. } \\
=\text { Scytinopogon angulisporus } \\
\text { =Clavaria atroumbrina } \\
\text { Corner }\end{array}$ & on soil & $\begin{array}{l}\text { Chambaghat, } \\
\text { Solan, Himachal } \\
\text { Pradesh }\end{array}$ & $\begin{array}{l}\text { Sharma and Munjal } \\
\text { (1977) }\end{array}$ \\
\hline 6. & Clavaria cretacea Coker & on soil & $\begin{array}{l}\text { Chambaghat, } \\
\text { Solan, Himachal } \\
\text { Pradesh }\end{array}$ & $\begin{array}{l}\text { Sharma and Munjal } \\
\text { (1977) }\end{array}$ \\
\hline
\end{tabular}




\begin{tabular}{|c|c|c|c|c|}
\hline 7. & Clavaria crosslandii Cotton & on soil & $\begin{array}{l}\text { Chambaghat, } \\
\text { Solan, Himachal } \\
\text { Pradesh }\end{array}$ & $\begin{array}{l}\text { Sharma and Munjal } \\
\text { (1977) }\end{array}$ \\
\hline 8. & $\begin{array}{l}\text { Clavaria fragilis Holmsk. } \\
=\text { Clavaria vermicularis var. } \\
\text { gracilis } \text { Bourdot \& Galzin }\end{array}$ & $\begin{array}{l}\text { on soil under oak } \\
\text { forest }\end{array}$ & $\begin{array}{l}\text { Mussoorie, } \\
\text { Uttarakhand }\end{array}$ & $\begin{array}{l}\text { Thind and Anand } \\
\text { (1956c) }\end{array}$ \\
\hline 9. & Clavaria gollanii Henn. & on ground & $\begin{array}{l}\text { Saharanpur, } \\
\text { Uttar Pradesh }\end{array}$ & $\begin{array}{l}\text { Butler and Bisby } \\
\text { (1931) }\end{array}$ \\
\hline 10. & Clavaria incarnata Weinm. & $\begin{array}{l}\text { on ground in } \\
\text { mosses under } \\
\text { mixed forest }\end{array}$ & $\begin{array}{l}\text { Darjeeling, } \\
\text { West Bengal }\end{array}$ & $\begin{array}{l}\text { Thind and Rattan } \\
\text { (1967) }\end{array}$ \\
\hline 11. & $\begin{array}{l}\text { Clavaria indica Corner, K.S. } \\
\text { Thind \& Dev }\end{array}$ & $\begin{array}{l}\text { on humus in oak } \\
\text { forest }\end{array}$ & $\begin{array}{l}\text { Mussoorie, } \\
\text { Uttarakhand }\end{array}$ & Corner et al., (1958) \\
\hline 12. & Clavaria jacquemontii Lév. & on ground & $\begin{array}{l}\text { Jammu and } \\
\text { Kashmir }\end{array}$ & Leveille (1844) \\
\hline 13. & Clavaria sp. & $\begin{array}{l}\text { on dead logs of } \\
\text { Tectona grandis, } \\
\text { Terminalia } \\
\text { tomentosa }\end{array}$ & $\begin{array}{l}\text { Kolhapur, } \\
\text { Maharashtra }\end{array}$ & Parndekar (1964) \\
\hline 14. & Clavaria vermicularis Scop. & $\begin{array}{l}\text { on soil under oak } \\
\text { forest }\end{array}$ & $\begin{array}{l}\text { Mussoorie, } \\
\text { Uttarakhand }\end{array}$ & $\begin{array}{l}\text { Thind and Anand } \\
\text { (1956c) }\end{array}$ \\
\hline 15. & $\begin{array}{l}\text { Clavaria versatilis (Quél.) } \\
\text { Sacc. \& Trotter =Ramaria } \\
\text { versatilis Quél. }\end{array}$ & on forest soil & $\begin{array}{l}\text { Nilambur, } \\
\text { Kerala }\end{array}$ & Mohanan (2011) \\
\hline 16. & Clavaria zollingeri Lév. & $\begin{array}{l}\text { on soil under oak } \\
\text { forest }\end{array}$ & $\begin{array}{l}\text { Mussoorie, } \\
\text { Uttarakhand }\end{array}$ & $\begin{array}{l}\text { Thind and Anand } \\
\text { (1956c) }\end{array}$ \\
\hline 17. & $\begin{array}{l}\text { Clavariadelphus } \\
\text { himalayensis Methven }\end{array}$ & $\begin{array}{l}\text { on soil under } \\
\text { Pinus insularis }\end{array}$ & Meghalaya & Methven (1989) \\
\hline 18. & $\begin{array}{l}\text { Clavariadelphus junceus } \\
\text { (Alb. \& Schwein.) Corner } \\
\equiv \text { Clavaria mira Pat. }\end{array}$ & on soil & $\begin{array}{l}\text { Mussoorie, } \\
\text { Uttarakhand }\end{array}$ & $\begin{array}{l}\text { Thind and Anand } \\
\text { (1956b) }\end{array}$ \\
\hline 19. & $\begin{array}{l}\text { Clavariadelphus truncatus } \\
\text { (Quél.) Donk =Clavaria } \\
\text { truncata Quél. } \\
=\text { Clavariadelphus borealis } \\
\text { V.L. Wells \& Kempton }\end{array}$ & on soil & $\begin{array}{l}\text { Mussoorie, } \\
\text { Uttarakhand }\end{array}$ & $\begin{array}{l}\text { Thind and Sukh Dev } \\
\text { (1956) }\end{array}$ \\
\hline 20. & $\begin{array}{l}\text { Clavulina amethystinoides } \\
\text { (Peck) Corner } \equiv \text { Clavaria } \\
\text { amethystinoides } \text { Peck }\end{array}$ & $\begin{array}{l}\text { on soil amid } \\
\text { mosses }\end{array}$ & $\begin{array}{l}\text { Mussooorie, } \\
\text { Uttarakhand }\end{array}$ & $\begin{array}{l}\text { Thind and Anand } \\
\text { (1956c) }\end{array}$ \\
\hline 21. & $\begin{array}{l}\text { Clavulina bessonii } \text { (Pat.) } \\
\text { Corner =Clavulina bessonii } \\
\text { var. incarnata } \text { K.S. Thind \& } \\
\text { Anand }\end{array}$ & on soil & $\begin{array}{l}\text { Mussoorie, } \\
\text { Uttarakhand }\end{array}$ & $\begin{array}{l}\text { Thind and Anand } \\
\text { (1956b) }\end{array}$ \\
\hline 22. & $\begin{array}{l}\text { Clavulina cartilaginea (Berk. } \\
\& \text { M.A. Curtis) Corner }\end{array}$ & $\begin{array}{l}\text { on soil amid } \\
\text { mosses under }\end{array}$ & $\begin{array}{l}\text { Mussoorie, } \\
\text { Uttarakhand }\end{array}$ & $\begin{array}{l}\text { Thind and Sukh Dev } \\
\text { (1956) }\end{array}$ \\
\hline
\end{tabular}




\begin{tabular}{|c|c|c|c|c|}
\hline & $\begin{array}{l}\equiv \text { Lachnocladium } \\
\text { cartilagineum Berk. \& M.A. } \\
\text { Curtis }\end{array}$ & Cedrus forest, & & \\
\hline 23. & $\begin{array}{l}\text { Clavulina cinerea (Bull.) J. } \\
\text { Schröt. =Clavulina cristata } \\
\text { (Fr.) Schroet. =Ramaria } \\
\text { cristata Holmsk. =Clavaria } \\
\text { cristata (Holmsk.) Pers. }\end{array}$ & $\begin{array}{l}\text { In Cynodon } \\
\text { dactylon and on } \\
\text { soil amid mosses } \\
\text { under oak forest }\end{array}$ & $\begin{array}{l}\text { Patharia forest, } \\
\text { Sagar, Madhya } \\
\text { Pradesh and } \\
\text { Mussoorie, } \\
\text { Uttarakhand }\end{array}$ & $\begin{array}{l}\text { Dehariya } \text { et al., } \\
\text { (2010); Thind and } \\
\text { Anand (1956c) }\end{array}$ \\
\hline 24. & $\begin{array}{l}\text { Clavulina coralloides }(\mathrm{L} .) \mathrm{J} \text {. } \\
\text { Schröt. = Clavulina cristata } \\
\text { var. brunneola } \text { K.S. Thind \& } \\
\text { Anand }\end{array}$ & $\begin{array}{l}\text { on soil, dead } \\
\text { leaves, twigs and } \\
\text { bark }\end{array}$ & $\begin{array}{l}\text { Mussoorie, } \\
\text { Uttarakhand }\end{array}$ & $\begin{array}{l}\text { Thind and Anand } \\
\text { (1956c) }\end{array}$ \\
\hline 25. & $\begin{array}{l}\text { Clavulina hispidulosa } \\
\text { Corner, K.S. Thind \& Anand }\end{array}$ & $\begin{array}{l}\text { on humus soil } \\
\text { under oak forest }\end{array}$ & $\begin{array}{l}\text { Mussoorie, } \\
\text { Uttarakhand }\end{array}$ & Corner et al., (1956) \\
\hline 26. & $\begin{array}{l}\text { Clavulina limosa K.S. Thind } \\
\text { \& Sharda }\end{array}$ & $\begin{array}{l}\text { on sandy soil } \\
\text { under Shorea } \\
\text { robusta }\end{array}$ & West Bengal & $\begin{array}{l}\text { Thind and Sharda } \\
\text { (1984) }\end{array}$ \\
\hline 27. & $\begin{array}{l}\text { Clavulina mussooriensis } \\
\text { Corner, K.S. Thind \& Dev }\end{array}$ & $\begin{array}{l}\text { on soil under } \\
\text { Cedrua forest }\end{array}$ & $\begin{array}{l}\text { Mussoorie, } \\
\text { Uttarakhand }\end{array}$ & Corner et al., (1958) \\
\hline 28. & $\begin{array}{l}\text { Clavulina ornatipes }(\text { Peck) } \\
\text { Corner }=\text { Lachnocladium } \\
\text { ornatipes }(\text { Peck) Burt } \\
\text { =Clavaria ornatipes }\end{array}$ & $\begin{array}{l}\text { on soil under oak } \\
\text { forest and } \\
\text { decaying leaves } \\
\text { on a side of pond }\end{array}$ & $\begin{array}{l}\text { Shimla, } \\
\text { Himachal } \\
\text { Pradesh and } \\
\text { Kolkata, West } \\
\text { Bengal }\end{array}$ & $\begin{array}{l}\text { Berkeley (1856); } \\
\text { Thind and Rattan } \\
\text { (1967) }\end{array}$ \\
\hline 29. & $\begin{array}{l}\text { Clavulina rugosa (Bull.) J. } \\
\text { Schröt. = Clavulina rugosa } \\
\text { var. alcyonaria Corner }\end{array}$ & on soil & $\begin{array}{l}\text { Chambaghat, } \\
\text { Solan, Himachal } \\
\text { Pradesh }\end{array}$ & $\begin{array}{l}\text { Sharma and Munjal } \\
\text { (1977) }\end{array}$ \\
\hline 30. & $\begin{array}{l}\text { Clavulina subrugosa } \\
\text { (Cleland) Corner } \equiv \text { Clavaria } \\
\text { subrugosa Cleland }\end{array}$ & $\begin{array}{l}\text { on soil amid } \\
\text { mosses }\end{array}$ & $\begin{array}{l}\text { Mussoorie, } \\
\text { Uttarakhand }\end{array}$ & $\begin{array}{l}\text { Thind and Raswan } \\
\text { (1958) }\end{array}$ \\
\hline 31. & $\begin{array}{l}\text { Clavulinopsis alcicornis } \\
\text { (Zoll. \& Moritzi) Corner } \\
\text { 三Clavaria alcicornis Zoll. \& } \\
\text { Moritzi }\end{array}$ & on soil & $\begin{array}{l}\text { Mussoorie, } \\
\text { Uttarakhand }\end{array}$ & $\begin{array}{l}\text { Thind and Anand } \\
\text { (1956b) }\end{array}$ \\
\hline 32. & $\begin{array}{l}\text { Clavulinopsis amoena } \text { (Zoll. } \\
\& \text { Moritzi) Corner } \equiv \text { Clavaria } \\
\text { amoena Zoll. \& Moritzi }\end{array}$ & on soil & $\begin{array}{l}\text { Dalhausi, } \\
\text { Himachal } \\
\text { Pradesh }\end{array}$ & $\begin{array}{l}\text { Thind and Rattan } \\
\text { (1967) }\end{array}$ \\
\hline 33. & $\begin{array}{l}\text { Clavulinopsis } \\
\text { aurantiocinnabarina } \\
\text { (Schwein.) Corner } \equiv \text { Clavaria } \\
\text { aurantiocinnabarina } \\
\text { Schwein. }\end{array}$ & on soil & $\begin{array}{l}\text { Mussoorie, } \\
\text { Uttarakhand }\end{array}$ & $\begin{array}{l}\text { Thind and Anand } \\
\text { (1956b) }\end{array}$ \\
\hline 34. & $\begin{array}{l}\text { Clavulinopsis corniculata } \\
\text { (Schaeff.) Corner } \equiv \text { Clavaria } \\
\text { muscoides } \text { L. = Ramaria }\end{array}$ & on soil & $\begin{array}{l}\text { Mussoorie, } \\
\text { Uttarakhand and } \\
\text { Sonamarg, }\end{array}$ & $\begin{array}{l}\text { Thind and Anand } \\
\text { (1956b); Butler and } \\
\text { Bisby (1931) }\end{array}$ \\
\hline
\end{tabular}




\begin{tabular}{|c|c|c|c|c|}
\hline & corniculata (Schaeff.) Gray & & $\begin{array}{l}\text { Jammu and } \\
\text { Kashmir }\end{array}$ & \\
\hline 35. & $\begin{array}{l}\text { Clavulinopsis dichotoma } \\
\text { Corner } \equiv \text { Clavaria dichotoma } \\
\text { Godey }\end{array}$ & on soil & $\begin{array}{l}\text { Mussoorie, } \\
\text { Uttarakhand }\end{array}$ & $\begin{array}{l}\text { Thind and Sukh Dev } \\
\text { (1957b) }\end{array}$ \\
\hline 36. & $\begin{array}{l}\text { Clavulinopsis fusiformis } \\
\text { (Sowerby) Corner } \equiv \text { Clavaria } \\
\text { fusiformis Sowerby } \\
=\text { Clavulinopsis fusiformis } \\
\text { var. bispora K.S. Thind \& } \\
\text { Sharda }\end{array}$ & on soil & $\begin{array}{l}\text { Mussoorie, } \\
\text { Uttarakhand } \\
\text { Himachal } \\
\text { Pradesh }\end{array}$ & $\begin{array}{l}\text { Butler and Bisby } \\
\text { (1931); } \\
\text { Thind and Sharda } \\
\text { (1982) }\end{array}$ \\
\hline 37. & $\begin{array}{l}\text { Clavulinopsis helvola (Pers.) } \\
\text { Corner }\end{array}$ & $\begin{array}{l}\text { on soil under oak } \\
\text { forest }\end{array}$ & $\begin{array}{l}\text { Brewery road, } \\
\text { Mussoorie }\end{array}$ & $\begin{array}{l}\text { Thind and Raswan } \\
\text { (1958) }\end{array}$ \\
\hline 38. & $\begin{array}{l}\text { Clavulinopsis laeticolor } \\
\text { (Berk. \& M.A. Curtis) R.H. } \\
\text { Petersen =Clavaria laeticolor } \\
\text { Berk. \& M.A. Curtis = } \\
\text { Clavulinopsis laeticolor f. } \\
\text { bispora K.S. Thind \& Sharda } \\
\text { =Clavulinopsis pulchra f. } \\
\text { subtrigona K.S. Thind \& S.S. } \\
\text { Rattan = Clavulinopsis } \\
\text { pulchra var. coccinea K.S. } \\
\text { Thind \& Dev }\end{array}$ & $\begin{array}{l}\text { on rotten timber in } \\
\text { wet woods, on soil } \\
\text { on decaying } \\
\text { leaves and in } \\
\text { broad-leaved } \\
\text { forest } \\
\text { on humicolous } \\
\text { soil under forest } \\
\text { on humicolous } \\
\text { soil }\end{array}$ & $\begin{array}{l}\text { Kala Pani, } \\
\text { Khasi hills, } \\
\text { Meghalaya } \\
\text { Chambaghat, } \\
\text { Solan, Himachal } \\
\text { Pradesh } \\
\text { Kolkata, West } \\
\text { Bengal } \\
\text { Darjeeling, } \\
\text { West Bengal } \\
\text { and } \\
\text { Chakarata, } \\
\text { Mussoorie, } \\
\text { Uttarakhand }\end{array}$ & $\begin{array}{l}\text { Berkeley (1852a) } \\
\text { Sharma and Munjal } \\
\text { (1977) } \\
\text { Thind and Sharda } \\
\text { (1986) (1987); } \\
\text { Banerjee (1947) } \\
\text { Thind and Rattan } \\
\text { (1967) } \\
\text { Thind and Dev } \\
\text { (1957) }\end{array}$ \\
\hline 39. & $\begin{array}{l}\text { Clavulinopsis semivestita } \\
\text { (Berk. \& Broome) Corner }\end{array}$ & on soil & $\begin{array}{l}\text { Chambaghat, } \\
\text { Solan, Himachal } \\
\text { Pradesh }\end{array}$ & $\begin{array}{l}\text { Sharma and Munjal } \\
\text { (1977) }\end{array}$ \\
\hline 40. & $\begin{array}{l}\text { Clavulinopsis subtilis (Pers.) } \\
\text { Corner }\end{array}$ & $\begin{array}{l}\text { on soil under oak } \\
\text { forest }\end{array}$ & $\begin{array}{l}\text { Mussoorie, } \\
\text { Uttarakhand }\end{array}$ & $\begin{array}{l}\text { Thind and Raswan } \\
\text { (1958) }\end{array}$ \\
\hline 41. & $\begin{array}{l}\text { Clavulinopsis sulcata } \\
\text { Overeem =Clavulinopsis } \\
\text { miniata Corner }\end{array}$ & on soil & $\begin{array}{l}\text { Darjeeling, } \\
\text { West Bengal }\end{array}$ & $\begin{array}{l}\text { Thind and Rattan } \\
\text { (1967) }\end{array}$ \\
\hline 42. & $\begin{array}{l}\text { Deflexula subsimplex (Henn.) } \\
\text { Corner } \equiv \text { Pterula subsimplex } \\
\text { Henn. }\end{array}$ & $\begin{array}{l}\text { on stump of } \\
\text { Cryptomeria } \\
\text { japonica }\end{array}$ & $\begin{array}{l}\text { Darjeeling, } \\
\text { West Bengal }\end{array}$ & $\begin{array}{l}\text { Thind and Rattan } \\
\text { (1967) }\end{array}$ \\
\hline 43. & $\begin{array}{l}\text { Gloeocantharellus lateritius } \\
\text { (Petch) Corner } \equiv \text { Paxillus } \\
\text { lateritius } \text { Petch }\end{array}$ & on soil & $\begin{array}{l}\text { Malappuram, } \\
\text { Kerala }\end{array}$ & $\begin{array}{l}\text { Joseph and } \\
\text { Manimohan, (1998) }\end{array}$ \\
\hline 44. & $\begin{array}{l}\text { Lachnocladium brasiliense } \\
\text { (Lév.) Pat. =Eriocladus } \\
\text { brasiliensis Lév., }\end{array}$ & $\begin{array}{l}\text { on earth and } \\
\text { decaying leaves }\end{array}$ & $\begin{array}{l}\text { Kolkata, West } \\
\text { Bengal }\end{array}$ & Berkeley (1856) \\
\hline 45. & $\begin{array}{l}\text { Lachnocladium hookeri } \\
\text { Berk. }\end{array}$ & - & $\begin{array}{l}\text { Khasi Hills, } \\
\text { Meghalaya }\end{array}$ & Berkeley (1852b) \\
\hline
\end{tabular}




\begin{tabular}{|c|c|c|c|c|}
\hline 46. & $\begin{array}{l}\text { Lachnocladium mussooriense } \\
\text { Henn. }\end{array}$ & - & $\begin{array}{l}\text { Arnigadh, } \\
\text { Mussoorie, } \\
\text { Uttarakhand }\end{array}$ & Hennings (1901) \\
\hline 47. & Lentaria byssiseda Corner & $\begin{array}{l}\text { on dead twigs of } \\
\text { Picea morinda in } \\
\text { forest }\end{array}$ & $\begin{array}{l}\text { Mussoorie, } \\
\text { Uttarakhand }\end{array}$ & $\begin{array}{l}\text { Thind and Sukh Dev } \\
\text { (1956) }\end{array}$ \\
\hline 48. & $\begin{array}{l}\text { Lentaria epichnoa }(\mathrm{Fr} .) \\
\text { Corner = Lentaria epichnoa } \\
\text { var. indica Sharda }\end{array}$ & on wood & Andhra Pradesh & Sharda (1984) \\
\hline 49. & $\begin{array}{l}\text { Multiclavula mucida } \text { (Pers.) } \\
\text { R.H. Petersen } \equiv \text { Clavaria } \\
\text { mucida Pers. =Lentaria } \\
\text { mucida } \text { (Pers.) Corner }\end{array}$ & $\begin{array}{l}\text { on decaying logs } \\
\text { of Picea morinda, } \\
\text { under Picea forest }\end{array}$ & $\begin{array}{l}\text { Kadukhal, } \\
\text { Mussoorie, } \\
\text { Uttarakhand }\end{array}$ & $\begin{array}{l}\text { Thind and Sukh Dev } \\
\text { (1957a) }\end{array}$ \\
\hline 50. & $\begin{array}{l}\text { Phaeoclavulina flaccida } \text { (Fr.) } \\
\text { Giachini =Ramaria flaccida } \\
\text { (Fr.) Bourdot =Clavaria } \\
\text { flaccida } \text { Fr., =Clavariella } \\
\text { flaccida } \text { (Fr.) P. Karst. }\end{array}$ & $\begin{array}{l}\text { on humus under } \\
\text { oak and pine } \\
\text { forests and on } \\
\text { living stem of } 12 \\
\text { years old teak }\end{array}$ & $\begin{array}{l}\text { Mussoorie, } \\
\text { Uttarakhand and } \\
\text { Jabalpur, } \\
\text { Madhya Pradesh }\end{array}$ & $\begin{array}{l}\text { Thind and Anand } \\
\text { (1956a) } \\
\text { This article }\end{array}$ \\
\hline 51. & $\begin{array}{l}\text { Phaeoclavulina zippelii } \\
\text { (Lév.) Overeem =Clavaria } \\
\text { zippelii Lév. }\end{array}$ & on ground & $\begin{array}{l}\text { Lingamala fall, } \\
\text { Maharashtra }\end{array}$ & Senthilarasu (2013a) \\
\hline 52. & Pterula indica Senthil. & $\begin{array}{l}\text { on soil in Acacia } \\
\text { and Dalbergia, } \\
\text { forest }\end{array}$ & $\begin{array}{l}\text { Pune, } \\
\text { Maharashtra }\end{array}$ & Senthilarasu (2013b) \\
\hline 53. & Pterula verticillata Corner & $\begin{array}{l}\text { on soil in Acacia } \\
\text { and forest }\end{array}$ & $\begin{array}{l}\text { Pune, } \\
\text { Maharashtra }\end{array}$ & Senthilarasu (2013b) \\
\hline 54. & $\begin{array}{l}\text { Ramaria apiculata (Fr.) } \\
\text { Donk }\end{array}$ & on soil & $\begin{array}{l}\text { Radhanagri, MS } \\
\text { and Ariappa, } \\
\text { Kerala }\end{array}$ & $\begin{array}{l}\text { Thite } \text { et al., (1976); } \\
\text { Patil and Thite } \\
\text { (1977); Mohanan } \\
\text { (2011) }\end{array}$ \\
\hline 55. & $\begin{array}{l}\text { Ramaria aurea (Schaeff.) } \\
\text { Quél. }\end{array}$ & $\begin{array}{l}\text { Rhododendron } \\
\text { forest }\end{array}$ & $\begin{array}{l}\text { Shimla, } \\
\text { Himachal } \\
\text { Pradesh and } \\
\text { Sikkim }\end{array}$ & $\begin{array}{l}\text { Sharma and Jandaik } \\
\text { (1978); Das (2009) }\end{array}$ \\
\hline 56. & $\begin{array}{l}\text { Ramaria brevispora Corner, } \\
\text { K.S. Thind \& Dev }\end{array}$ & $\begin{array}{l}\text { on soil in oak } \\
\text { forest }\end{array}$ & $\begin{array}{l}\text { Mussoorie, } \\
\text { Uttarakhand and } \\
\text { Sikkim }\end{array}$ & $\begin{array}{l}\text { Corner } \text { et al., (1958); } \\
\text { Das (2009) }\end{array}$ \\
\hline 57. & $\begin{array}{l}\text { Ramaria camelicolor Corner, } \\
\text { K.S. Thind \& Anand }\end{array}$ & $\begin{array}{l}\text { on soil in oak } \\
\text { forest, on soil in } \\
\text { forest }\end{array}$ & $\begin{array}{l}\text { Mussoorie, } \\
\text { Uttarakhand and } \\
\text { Shergaon, } \\
\text { Arunachal } \\
\text { Pradesh }\end{array}$ & $\begin{array}{l}\text { Corner et al., (1956) } \\
\text { Sharda and Thind } \\
\text { (1986) }\end{array}$ \\
\hline 58. & $\begin{array}{l}\text { Ramaria clarobrunnea } \\
\text { Corner, K.S. Thind \& Anand }\end{array}$ & $\begin{array}{l}\text { on soil in oak } \\
\text { forest }\end{array}$ & $\begin{array}{l}\text { Mussoorie, } \\
\text { Uttarakhand }\end{array}$ & Corner et al., (1956) \\
\hline 59. & Ramaria concolor (Corner) & on wood, on & Mussoorie, & Thind and Sukh Dev \\
\hline
\end{tabular}




\begin{tabular}{|c|c|c|c|c|}
\hline & $\begin{array}{l}\text { R.H. Petersen = Ramaria } \\
\text { stricta } \text { (Pers.) Quél. var. } \\
\text { concolor Corner }\end{array}$ & $\begin{array}{l}\text { stumps and logs, } \\
\text { on dead leaves in } \\
\text { oak forest }\end{array}$ & $\begin{array}{l}\text { Uttarakhand and } \\
\text { Shimla, } \\
\text { Himachal } \\
\text { Pradesh }\end{array}$ & $\begin{array}{l}\text { (1957a b); Sharma } \\
\text { and Jandaik (1978) }\end{array}$ \\
\hline 60. & $\begin{array}{l}\text { Ramaria echinovirens } \\
\text { Corner, K.S. Thind \& Dev }\end{array}$ & $\begin{array}{l}\text { on soil in oak } \\
\text { forest }\end{array}$ & $\begin{array}{l}\text { Mussoorie, } \\
\text { Uttarakhand }\end{array}$ & Corner et al., (1957) \\
\hline 61. & $\begin{array}{l}\text { Ramaria eumorpha }(\mathrm{P} . \\
\text { Karst.) Corner } \equiv \text { Clavariella } \\
\text { eumorpha } \mathrm{P} . \text { Karst. }\end{array}$ & on soil & $\begin{array}{l}\text { Nilambur, } \\
\text { Kerala and } \\
\text { Gulmerg, } \\
\text { Jammu \& } \\
\text { Kashmir }\end{array}$ & $\begin{array}{l}\text { Mohanan (2011); } \\
\text { Thind et al., (1983) }\end{array}$ \\
\hline 62. & $\begin{array}{l}\text { Ramaria flava (Schaeff.) } \\
\text { Quél. = Ramaria flava var. } \\
\text { sanguine }\end{array}$ & on soil and humus & $\begin{array}{l}\text { Mussoorie, } \\
\text { Uttarakhand } \\
\text { and Nilambur, } \\
\text { Kerala }\end{array}$ & $\begin{array}{l}\text { Corner (1956) } \\
\text { Thind and Sukh Dev } \\
\text { (1957a); Mohanan } \\
\text { (2011) }\end{array}$ \\
\hline 63. & $\begin{array}{l}\text { Ramaria flaviceps Corner, } \\
\text { K.S. Thind \& Anand }\end{array}$ & $\begin{array}{l}\text { on soil in oak } \\
\text { forest }\end{array}$ & $\begin{array}{l}\text { Mussoorie, } \\
\text { Uttarakhand }\end{array}$ & Corner et al., (1956) \\
\hline 64. & Ramaria flavoalba Corner & in wood land & $\begin{array}{l}\text { Solan, Himachal } \\
\text { Pradesh }\end{array}$ & Sharma et al., (1977) \\
\hline 65. & $\begin{array}{l}\text { Ramaria flavobrunnescens } \\
\text { (Coker) Corner }\end{array}$ & $\begin{array}{l}\text { on soil amid } \\
\text { mosses under oak } \\
\text { forest }\end{array}$ & $\begin{array}{l}\text { Mussoorie, } \\
\text { Uttarakhand and } \\
\text { West Kameng, } \\
\text { Arunachal } \\
\text { Pradesh }\end{array}$ & $\begin{array}{l}\text { Thind and Sukh Dev } \\
\text { (1957a) and Sharda } \\
\text { and Thind (1986) }\end{array}$ \\
\hline 66. & $\begin{array}{l}\text { Ramaria flavoviridis Corner } \\
\& \text { K.S. Thind }\end{array}$ & $\begin{array}{l}\text { on ground in } \\
\text { mixed Quercus- } \\
\text { Cedrus forest }\end{array}$ & $\begin{array}{l}\text { Bakrota, } \\
\text { Dalhaousie, } \\
\text { Himachal } \\
\text { Pradesh }\end{array}$ & $\begin{array}{l}\text { Corner and Thind } \\
\text { (1961) }\end{array}$ \\
\hline 67. & $\begin{array}{l}\text { Ramaria formosa } \text { (Pers.) } \\
\text { Quél. =Clavaria formosa } \\
\text { Pers. }\end{array}$ & $\begin{array}{l}\text { on soil and humus } \\
\text { and } \\
\text { on soil under oak } \\
\text { forest }\end{array}$ & $\begin{array}{l}\text { Khasi hills, } \\
\text { Meghalaya; } \\
\text { Nilambur, } \\
\text { Kerala and } \\
\text { Mussoorie, } \\
\text { Uttarakhand }\end{array}$ & $\begin{array}{l}\text { Berkeley (1856); } \\
\text { Mohanan (2011); } \\
\text { Thind and Anand } \\
\text { (1956c) }\end{array}$ \\
\hline 68. & $\begin{array}{l}\text { Ramaria gracilis (Pers.) } \\
\text { Quél. = Clavaria gracilis } \\
\text { Pers. }\end{array}$ & on soil and humus & $\begin{array}{l}\text { Kandaghat, } \\
\text { Himachal } \\
\text { Pradesh and } \\
\text { Peechi, Kerala }\end{array}$ & $\begin{array}{l}\text { Sharma and Jandaik } \\
\text { (1978); Mohanan } \\
\text { (2011) }\end{array}$ \\
\hline 69. & $\begin{array}{l}\text { Ramaria holorubella (G.F. } \\
\text { Atk.) Corner =Clavaria } \\
\text { holorubella G.F. Atk. }\end{array}$ & - & $\begin{array}{l}\text { Ranikhet, } \\
\text { Uttarakhand and } \\
\text { Dalhausi, } \\
\text { Himachal } \\
\text { Pradesh }\end{array}$ & $\begin{array}{l}\text { Thind and Rattan } \\
\text { (1967) }\end{array}$ \\
\hline 70. & $\begin{array}{l}\text { Ramaria kisantuensis (Sacc.) } \\
\text { Corner = Ramaria } \\
\text { kisantuensis } \text { var. indica }\end{array}$ & on wood & Uttarakhand & $\begin{array}{l}\text { Khurana and Thind } \\
\text { (1979) }\end{array}$ \\
\hline
\end{tabular}




\begin{tabular}{|c|c|c|c|c|}
\hline & Khurana \& K.S. Thind & & & \\
\hline 71. & $\begin{array}{l}\text { Ramaria laevispora Corner } \\
\& \text { K.S. Thind }\end{array}$ & - & $\begin{array}{l}\text { Dalhousie, } \\
\text { Himachal } \\
\text { Pradesh }\end{array}$ & in Corner (1966) \\
\hline 72. & $\begin{array}{l}\text { Ramaria moelleriana (Bres. } \\
\& \text { Roum.) Corner }\end{array}$ & on soil & $\begin{array}{l}\text { Mussoorie, } \\
\text { Uttarakhand }\end{array}$ & $\begin{array}{l}\text { Thind and Sukh Dev } \\
\text { (1957b) }\end{array}$ \\
\hline 73. & $\begin{array}{l}\text { Ramaria obtusissima (Peck) } \\
\text { Corner }\end{array}$ & $\begin{array}{l}\text { on soil under oak, } \\
\text { Cedrus and pine } \\
\text { forests }\end{array}$ & $\begin{array}{l}\text { Mussoorie, } \\
\text { Uttarakhand and } \\
\text { Shillong, } \\
\text { Meghalaya }\end{array}$ & $\begin{array}{l}\text { Thind and Sukh Dev } \\
\text { (1957a b) and Sharda } \\
\text { and Thind (1986) }\end{array}$ \\
\hline 74. & $\begin{array}{l}\text { Ramaria ochrochlora Furrer- } \\
\text { Ziogas \& Schild }\end{array}$ & - & $\begin{array}{l}\text { Gulmerg, } \\
\text { Jammu \& } \\
\text { Kashmir }\end{array}$ & Thind et al., (1983) \\
\hline 75. & $\begin{array}{l}\text { Ramaria pallida (Schaeff.) } \\
\text { Ricken } \equiv \text { Clavaria pallida } \\
\text { Schaeff. }\end{array}$ & $\begin{array}{l}\text { On humus rich } \\
\text { soil }\end{array}$ & Sholayar, Kerala & Mohanan (2011) \\
\hline 76. & $\begin{array}{l}\text { Ramaria perbrunnea Corner } \\
\& \text { K.S. Thind }\end{array}$ & in Quercus forest & $\begin{array}{l}\text { Dalhousie, } \\
\text { Himachal } \\
\text { Pradesh }\end{array}$ & in Corner (1966) \\
\hline 77. & $\begin{array}{l}\text { Ramaria petersenii K.S. } \\
\text { Thind \& Sharda }\end{array}$ & $\begin{array}{l}\text { in coniferous } \\
\text { wood }\end{array}$ & $\begin{array}{l}\text { Himachal } \\
\text { Pradesh }\end{array}$ & $\begin{array}{l}\text { Thind and Sharda } \\
\text { (1984) }\end{array}$ \\
\hline 78. & $\begin{array}{l}\text { Ramaria pura Corner \& K.S. } \\
\text { Thind }\end{array}$ & in Quercus forest & $\begin{array}{l}\text { Dalhousie, } \\
\text { Himachal } \\
\text { Pradesh }\end{array}$ & in Corner (1966) \\
\hline 79. & $\begin{array}{l}\text { Ramaria purpurissima } \text { R.H. } \\
\text { Petersen \& Scates } \equiv \text { Ramaria } \\
\text { fumigata var. gigantea } \text { K.S. } \\
\text { Thind \& Anand }\end{array}$ & on soil & $\begin{array}{l}\text { Mussoorie, } \\
\text { Uttarakhand }\end{array}$ & $\begin{array}{l}\text { Thind and Anand } \\
\text { (1956a) }\end{array}$ \\
\hline 80. & Ramaria pusilla Corner & $\begin{array}{l}\text { on soil under oak } \\
\text { forest and on dead } \\
\text { needles of Cedrus } \\
\text { forest }\end{array}$ & $\begin{array}{l}\text { Mussoorie, } \\
\text { Uttarakhand }\end{array}$ & $\begin{array}{l}\text { Thind and Sukh Dev } \\
\text { (1957b) }\end{array}$ \\
\hline 81. & $\begin{array}{l}\text { Ramaria rasilispora Marr \& } \\
\text { D.E. Stuntz }\end{array}$ & $\begin{array}{l}\text { on soil under } \\
\text { hardwoods }\end{array}$ & $\begin{array}{l}\text { Jamiri- } \\
\text { Buragaon, } \\
\text { Arunachal } \\
\text { Pradesh }\end{array}$ & $\begin{array}{l}\text { Sharda and Thind } \\
\text { (1986) }\end{array}$ \\
\hline 82. & $\begin{array}{l}\text { Ramaria rubrogelatinosa } \\
\text { Corner \& K.S. Thind }\end{array}$ & - & $\begin{array}{l}\text { Dalhousie, } \\
\text { Himachal } \\
\text { Pradesh and } \\
\text { Mussoorie, } \\
\text { Uttarakhand }\end{array}$ & in Corner (1966) \\
\hline 83. & $\begin{array}{l}\text { Ramaria sandaracina Marr } \\
\text { \& D.E. Stuntz }\end{array}$ & $\begin{array}{l}\text { on soil under } P \text {. } \\
\text { kesiya forest }\end{array}$ & $\begin{array}{l}\text { Elephant falls, } \\
\text { Meghalaya and } \\
\text { West Kameng, } \\
\text { Arunachal }\end{array}$ & $\begin{array}{l}\text { Sharda and Thind } \\
\text { (1986) }\end{array}$ \\
\hline
\end{tabular}




\begin{tabular}{|c|c|c|c|c|}
\hline & & & Pradesh & \\
\hline 84. & $\begin{array}{l}\text { Ramaria sanguinea (Coker) } \\
\text { Corner }\end{array}$ & on moist soil & $\begin{array}{l}\text { Mussoorie, } \\
\text { Uttarakhand }\end{array}$ & $\begin{array}{l}\text { Thind and Sukh Dev } \\
\text { (1957b) }\end{array}$ \\
\hline 85. & $\begin{array}{l}\text { Ramaria sikkimia S.S. Rattan } \\
\& \text { Khurana }\end{array}$ & on forest litter & $\begin{array}{l}\text { Darjeeling, } \\
\text { West Bengal }\end{array}$ & $\begin{array}{l}\text { Rattan and Khurana } \\
\text { (1978) }\end{array}$ \\
\hline 86. & $\begin{array}{l}\text { Ramaria stricta } \text { (Pers.) Quél. } \\
=\text { Clavaria stricta Pers. }\end{array}$ & - & $\begin{array}{l}\text { Khasi hills, } \\
\text { Meghalaya }\end{array}$ & Berkeley (1856) \\
\hline 87. & $\begin{array}{l}\text { Ramaria subalpina K. Das \& } \\
\text { K. Acharya }\end{array}$ & $\begin{array}{l}\text { on soil, associated } \\
\text { with Abies densa }\end{array}$ & $\begin{array}{l}\text { Sikkim, } \\
\text { Himalaya }\end{array}$ & Das et al., (2016) \\
\hline 88. & $\begin{array}{l}\text { Ramaria subaurantiaca } \\
\text { Corner }\end{array}$ & $\begin{array}{l}\text { on soil amid } \\
\text { mosses }\end{array}$ & $\begin{array}{l}\text { Mussoorie, } \\
\text { Uttarakhand }\end{array}$ & $\begin{array}{l}\text { Thind and Sukh Dev } \\
\text { (1957b) }\end{array}$ \\
\hline 89. & $\begin{array}{l}\text { Ramaria subbotrytis (Coker) } \\
\text { Corner }\end{array}$ & $\begin{array}{l}\text { on soil under oak } \\
\text { forest }\end{array}$ & $\begin{array}{l}\text { Mussoorie, } \\
\text { Uttarakhand }\end{array}$ & $\begin{array}{l}\text { Thind and Anand } \\
\text { (1956a) }\end{array}$ \\
\hline 90. & $\begin{array}{l}\text { Ramaria subgelatinosa } \\
\text { Corner }\end{array}$ & on soil & $\begin{array}{l}\text { Mussoorie, } \\
\text { Uttarakhand }\end{array}$ & $\begin{array}{l}\text { Thind and Sukh Dev } \\
\text { (1957b) }\end{array}$ \\
\hline 91. & Ramaria suecica (Fr.) Donk & $\begin{array}{l}\text { on humicolous } \\
\text { soil or leaf-litter } \\
\text { under angiosperm } \\
\text { forest }\end{array}$ & $\begin{array}{l}\text { West Kameng, } \\
\text { Arunachal } \\
\text { Pradesh }\end{array}$ & $\begin{array}{l}\text { Sharda and Thind } \\
\text { (1986) }\end{array}$ \\
\hline 92. & $\begin{array}{l}\text { Ramaria synaptopoda Marr } \\
\& \text { D.E. Stuntz }\end{array}$ & $\begin{array}{l}\text { on soil under } \\
\text { Pinus kesiya }\end{array}$ & $\begin{array}{l}\text { Royle forest } \\
\text { Shillong, } \\
\text { Meghalaya }\end{array}$ & $\begin{array}{l}\text { Sharda and Thind } \\
\text { (1986) }\end{array}$ \\
\hline 93. & $\begin{array}{l}\text { Ramariopsis asterella (G.F. } \\
\text { Atk.) Corner } \equiv \text { Clavaria } \\
\text { asterella G.F. Atk. }\end{array}$ & on ground & $\begin{array}{l}\text { Sodepur, West } \\
\text { Bengal }\end{array}$ & $\begin{array}{l}\text { Banerjee and } \\
\text { Ganguly (1945) }\end{array}$ \\
\hline 94. & $\begin{array}{l}\text { Ramariopsis biformis (G.F. } \\
\text { Atk.) R.H. Petersen } \\
\text { =Clavulinopsis biformis var. } \\
\text { elongata K.S. Thind \& } \\
\text { Anand }\end{array}$ & on humus soil & $\begin{array}{l}\text { Mussoorie, } \\
\text { Uttarakhand }\end{array}$ & $\begin{array}{l}\text { Thind and Anand } \\
\text { (1956b) }\end{array}$ \\
\hline 95. & $\begin{array}{l}\text { Ramariopsis kunzei (Fr.) } \\
\text { Corner }\end{array}$ & $\begin{array}{l}\text { in humicolous soil } \\
\text { under oak forest }\end{array}$ & $\begin{array}{l}\text { Mussoorie, } \\
\text { Uttarakhand and } \\
\text { Chikhaldara, } \\
\text { Maharashtra }\end{array}$ & $\begin{array}{l}\text { Thind and Sukh Dev } \\
\text { (1957a) and This } \\
\text { article }\end{array}$ \\
\hline 96. & $\begin{array}{l}\text { Ramariopsis subtilis (Pers.) } \\
\text { R.H. Petersen }\end{array}$ & $\begin{array}{l}\text { in wood residues } \\
\text { and on humus } \\
\text { soil, } \\
\text { under Pongamia } \\
\text { pinnata }\end{array}$ & $\begin{array}{l}\text { Marwahi, } \\
\text { Chhattusgarh, } \\
\text { Jabalpur } \\
\text { Madhya Pradesh }\end{array}$ & $\begin{array}{l}\text { Tiwari et al., (2013) } \\
\text { This article }\end{array}$ \\
\hline 97. & $\begin{array}{l}\text { Ramariopsis tenuicula } \\
\text { (Bourdot \& Galzin) R.H. } \\
\text { Petersen =Clavulinopsis } \\
\text { tenuicula (Bourdot \& Galzin) } \\
\text { Corner } \equiv \text { Clavaria tenuicula } \\
\text { Bourdot \& Galzin }\end{array}$ & $\begin{array}{l}\text { on soil and on } \\
\text { decaying and on } \\
\text { rotting leaves of } \\
\text { dead mosses } \\
\text { under oak forest }\end{array}$ & $\begin{array}{l}\text { Chambaghat, } \\
\text { Solan, Himachal } \\
\text { Pradesh and } \\
\text { Mussoorie, } \\
\text { Uttarakhand }\end{array}$ & $\begin{array}{l}\text { Sharma and Munjal } \\
\text { (1977); Thind and } \\
\text { Raswan (1958) }\end{array}$ \\
\hline
\end{tabular}




\begin{tabular}{|c|c|c|c|c|}
\hline 98. & $\begin{array}{l}\text { Scytinopogon angulisporus } \\
\text { (Pat.) Corner } \equiv \text { Clavaria } \\
\text { angulispora } \text { Pat. }\end{array}$ & on ground & $\begin{array}{l}\text { Kolkata, } \\
\text { Burdwan, } \\
\text { Rampurhat, } \\
\text { West Bengal }\end{array}$ & $\begin{array}{l}\text { Banerjee (1947); De } \\
\text { (1991) }\end{array}$ \\
\hline 99. & $\begin{array}{l}\text { Typhula himalayana (Corner) } \\
\text { Khurana } \equiv \text { Pistillaria } \\
\text { himalayana } \text { Corner }\end{array}$ & $\begin{array}{l}\text { on dead leaves } \\
\text { and stems of } \\
\text { Aconitum sp. }\end{array}$ & $\begin{array}{l}\text { Mussoorie, } \\
\text { Uttarakhand and } \\
\text { Darjeeling, } \\
\text { West Bengal }\end{array}$ & Khurana (1980) \\
\hline 100. & $\begin{array}{l}\text { Typhula longispora Corner, } \\
\text { K.S. Thind \& Dev }\end{array}$ & $\begin{array}{l}\text { on fern leaflets } \\
\text { and rachis }\end{array}$ & $\begin{array}{l}\text { Mussoorie, } \\
\text { Uttarakhand }\end{array}$ & Corner et al., (1957) \\
\hline 101. & $\begin{array}{l}\text { Typhula micans } \text { (Pers.) } \\
\text { Berthier =Clavaria micans } \\
\text { Pers. =Pistillaria granulata } \\
\text { Pat. }\end{array}$ & $\begin{array}{l}\text { on dead leaves of } \\
\text { compost }\end{array}$ & $\begin{array}{l}\text { Mussoorie, } \\
\text { Uttarakhand }\end{array}$ & $\begin{array}{l}\text { Thind and Sukh Dev } \\
\text { (1956) }\end{array}$ \\
\hline 102. & Typhula ovata P. Karst. & $\begin{array}{l}\text { Cautlea lutea, on } \\
\text { decayed leaves of } \\
\text { Pteris cratica and } \\
\text { a grass }\end{array}$ & $\begin{array}{l}\text { Mussoorie, } \\
\text { Uttarakhand }\end{array}$ & $\begin{array}{l}\text { Thind and Sukh Dev } \\
\text { (1957a) }\end{array}$ \\
\hline 103. & $\begin{array}{l}\text { Typhula phacorrhiza } \\
\text { (Reichard) Fr. } \equiv \text { Clavaria } \\
\text { phacorrhiza Reichard }\end{array}$ & $\begin{array}{l}\text { on decaying } \\
\text { leaves }\end{array}$ & $\begin{array}{l}\text { Kullu and } \\
\text { Langri, } \\
\text { Himachal } \\
\text { Pradesh }\end{array}$ & Khurana (1980) \\
\hline 104. & Typhula pteridicola Khurana & $\begin{array}{l}\text { on decaying } \\
\text { fronds of } \\
\text { Pteridophyta }\end{array}$ & $\begin{array}{l}\text { Himachal } \\
\text { Pradesh }\end{array}$ & Khurana (1980) \\
\hline 105. & Typhula pulgensis Khurana & $\begin{array}{l}\text { on decaying } \\
\text { leaves }\end{array}$ & $\begin{array}{l}\text { Himachal } \\
\text { Pradesh }\end{array}$ & Khurana (1980) \\
\hline 106. & $\begin{array}{l}\text { Typhula setipes (Grev.) } \\
\text { Berthier } \equiv \text { Pistillaria setipes } \\
\text { Grev. }\end{array}$ & $\begin{array}{l}\text { on dead and } \\
\text { decaying leaves } \\
\text { under the thick } \\
\text { shade }\end{array}$ & $\begin{array}{l}\text { Mussoorie, } \\
\text { Uttarakhand }\end{array}$ & $\begin{array}{l}\text { Thind and Raswan } \\
\text { (1958) }\end{array}$ \\
\hline 107. & Typhula thindii Khurana & $\begin{array}{l}\text { on stem and } \\
\text { foliage of } \\
\text { Hedychium } \\
\text { acuminatum }\end{array}$ & $\begin{array}{l}\text { Tiffon's top, } \\
\text { Nainital } \\
\text { Uttarakhand }\end{array}$ & Khurana (1980) \\
\hline 108. & $\begin{array}{l}\text { Typhula sclerotioides (Pers.) } \\
\text { Fr. }\end{array}$ & $\begin{array}{l}\text { On decaying } \\
\text { leaves and stem of } \\
\text { herbaceous plants }\end{array}$ & $\begin{array}{l}\text { Pulga, Kullu, } \\
\text { Himachal } \\
\text { Pradesh }\end{array}$ & Khurana (1980) \\
\hline
\end{tabular}



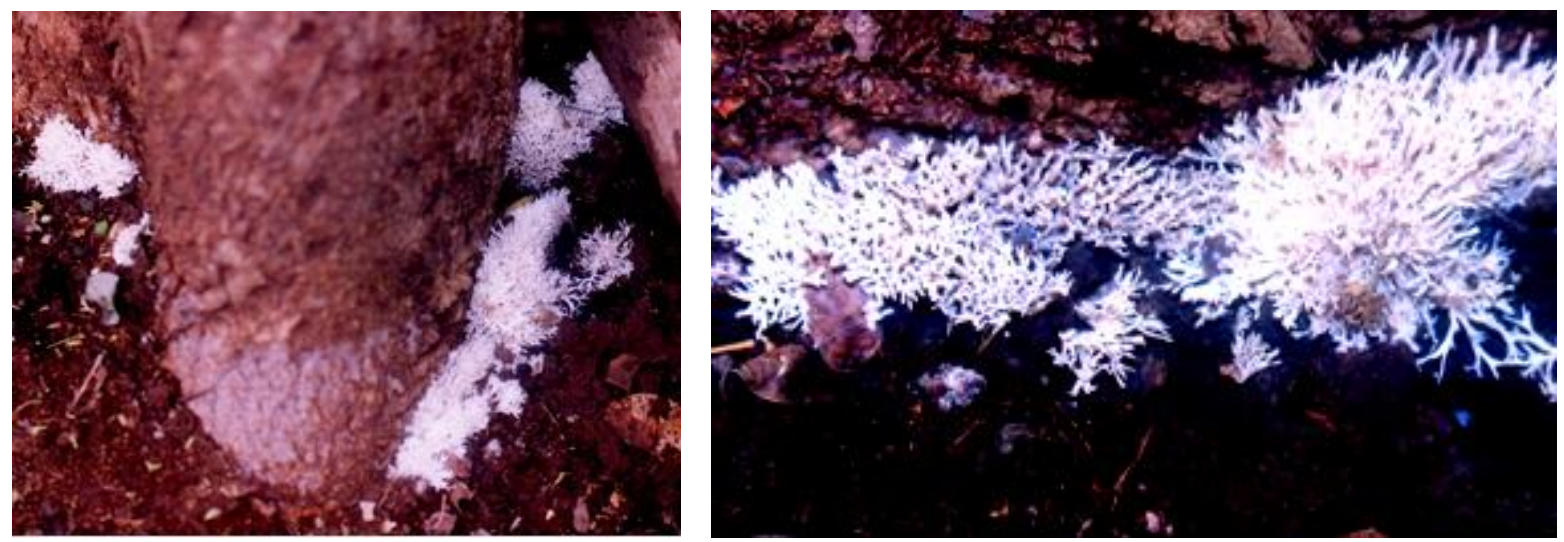

Figs.1-2 Phaeoclavulina flacida, habit, fruit bodies attached to living stem of 12 years old teak tree
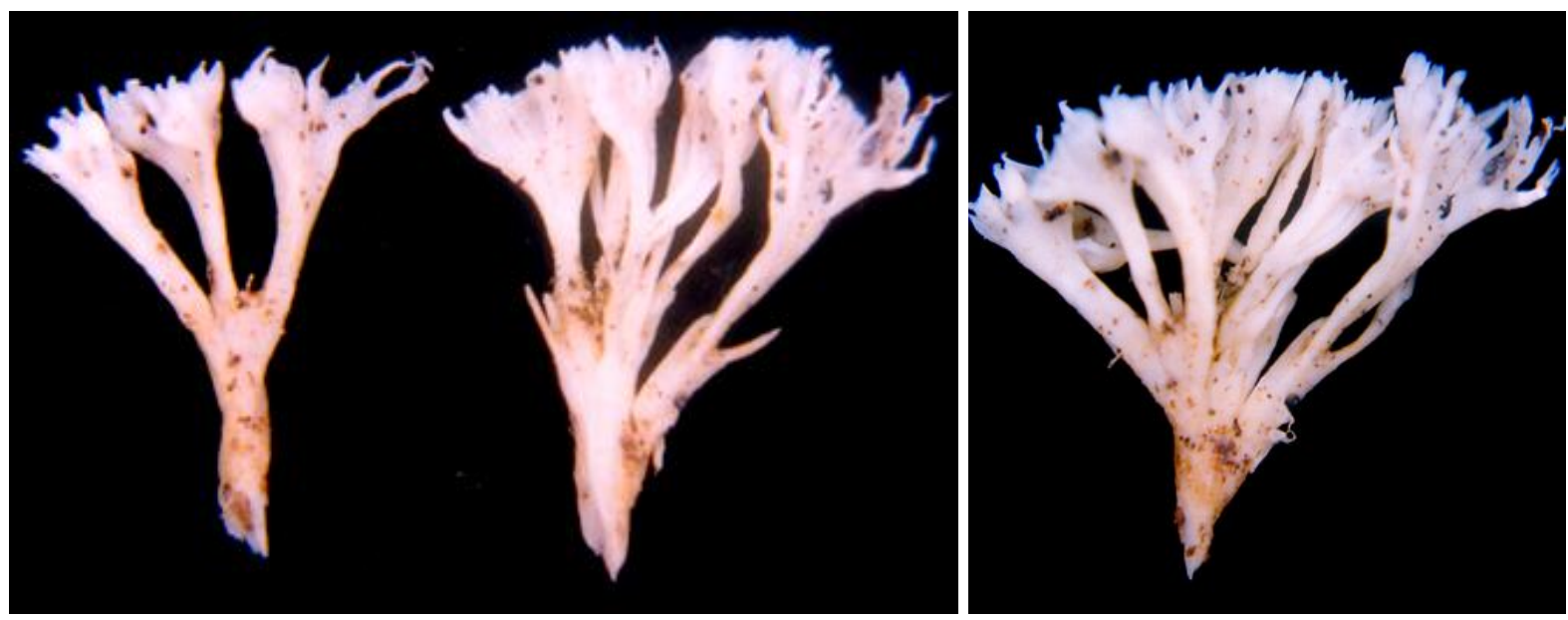

Figs. 3-4. Phaeoclavulina flaccida. Fruit bodied detached from living stem of 12 years old teak tree
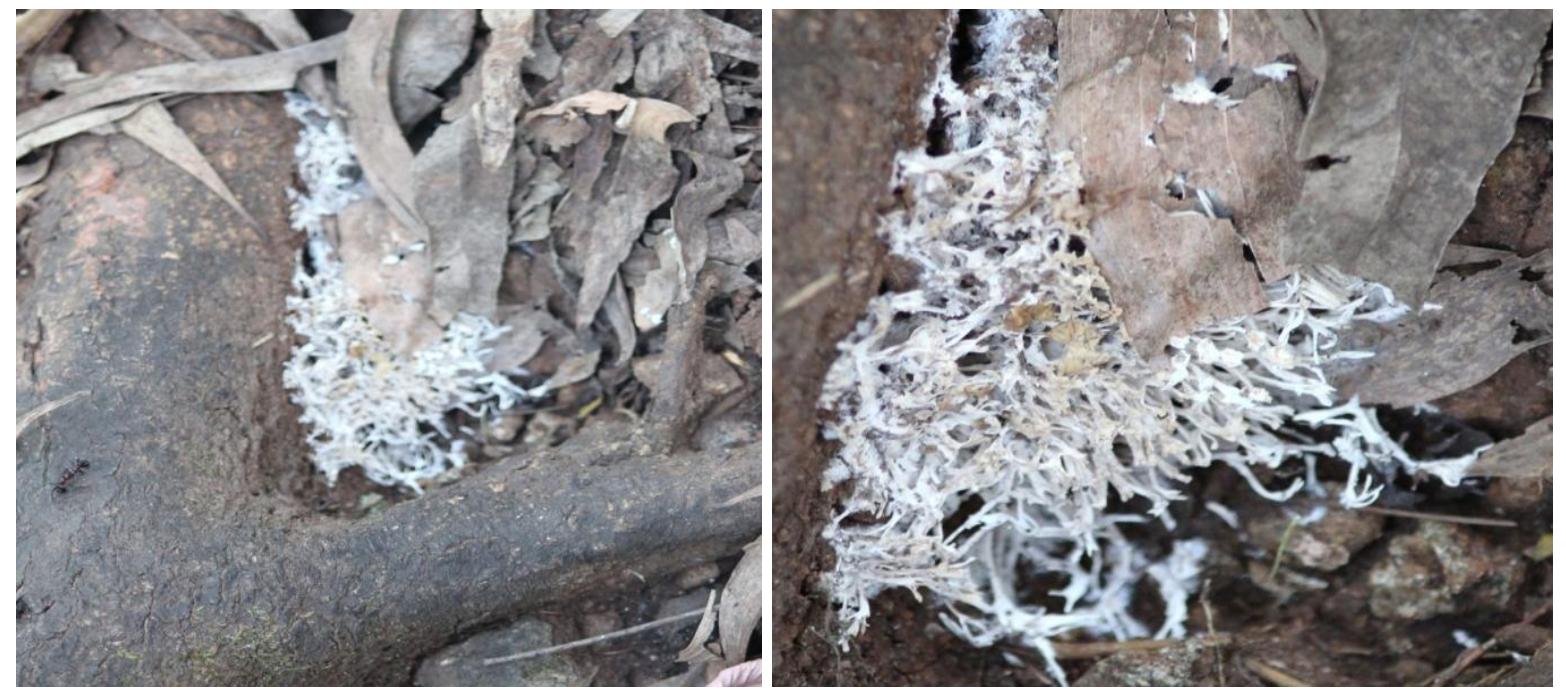

Figures 5-6 Ramariopsis kunzei: habit 

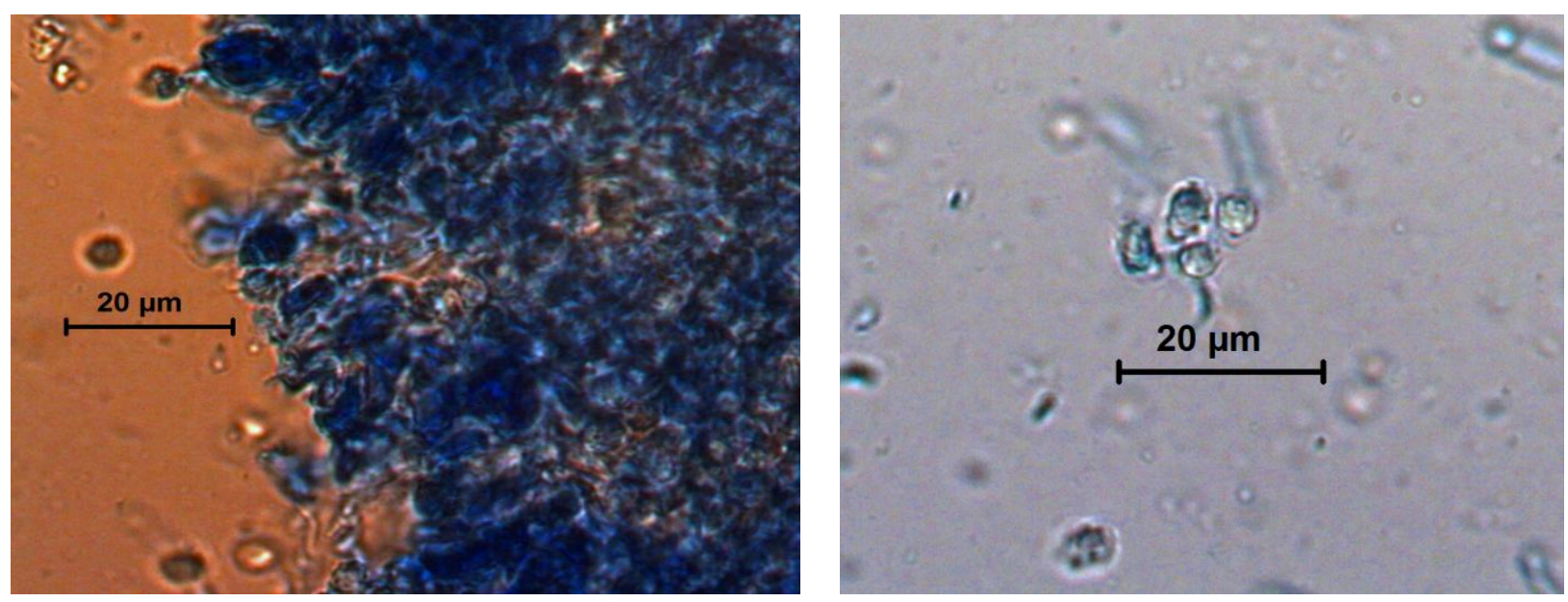

Figures 7-8 Ramariopsis kunzei: 7 basidia and other tissue, 8 basidiospores

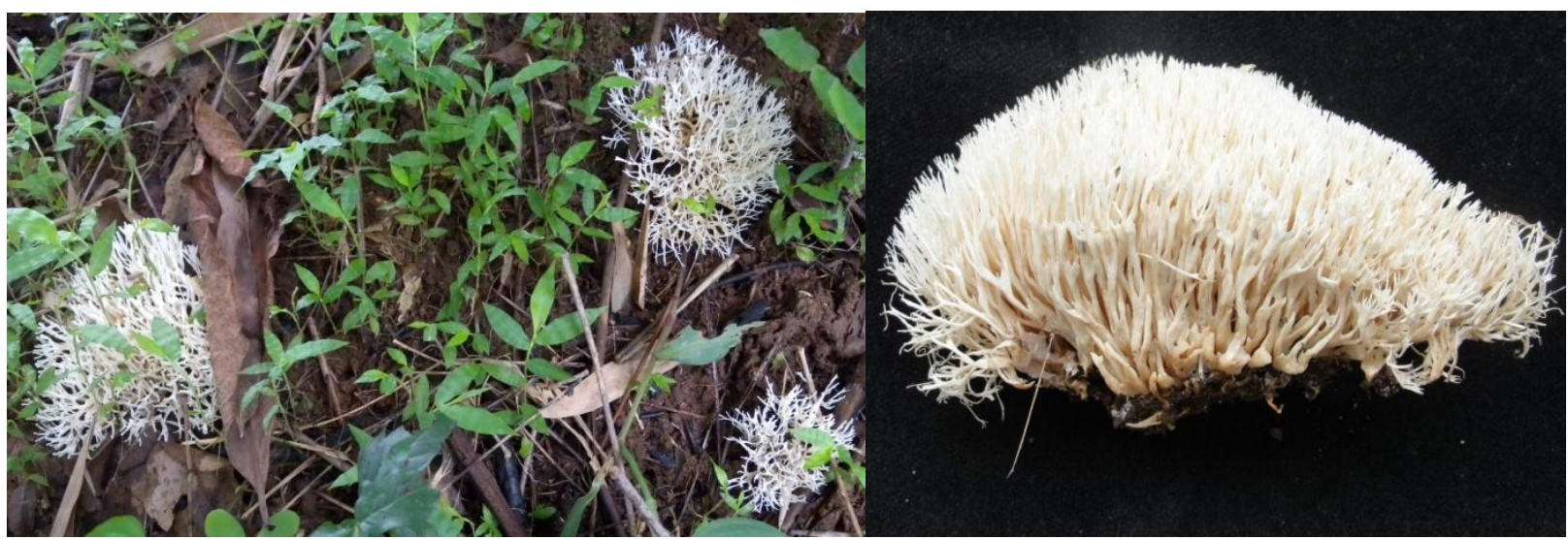

Figs. 9-10 Ramariopsis subtilis, 9 habit, 10 fruit body

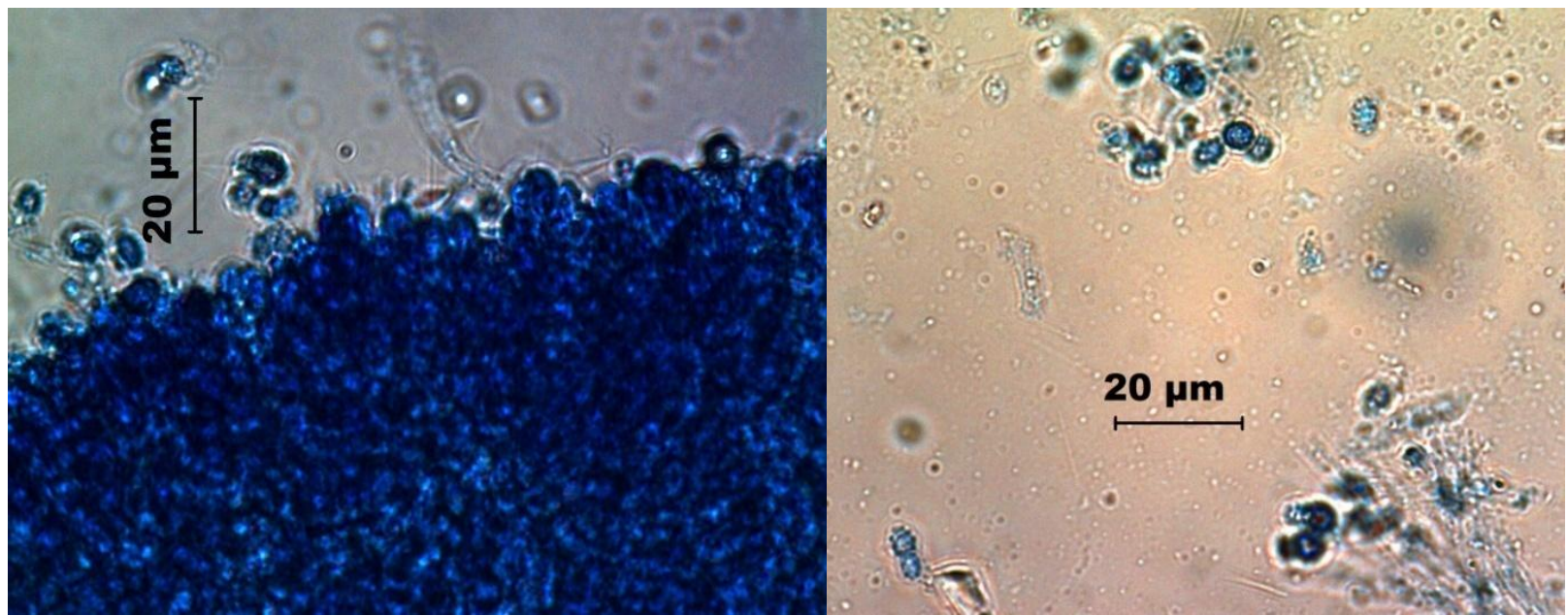

Fig. 11-12 Ramariopsis subtilis: 11 cross section showing basidia and 12 basidiospores 
Table.2 Distribution of clavarioid fungi in India

\begin{tabular}{|l|l|c|c|c|c|c|c|c|c|c|c|c|c|c|c|}
\hline S. & Genera & $\mathbf{( 1 )}$ & $\mathbf{( 2 )}$ & $\mathbf{( 3 )}$ & $\mathbf{( 4 )}$ & $\mathbf{( 5 )}$ & $\mathbf{( 6 )}$ & $\mathbf{( 7 )}$ & $\mathbf{( 8 )}$ & $\mathbf{( 9 )}$ & $\mathbf{( 1 0 )}$ & $\mathbf{( 1 1 )}$ & $\mathbf{( 1 2 )}$ & $\mathbf{( 1 3 )}$ & Total \\
N. & & $\mathbf{U K}$ & $\mathbf{H P}$ & $\mathbf{J K}$ & $\mathbf{M e g}$ & $\mathbf{M P}$ & $\mathbf{M S}$ & $\mathbf{W B}$ & $\mathbf{U P}$ & $\mathbf{A P}$ & $\mathbf{K e r}$ & $\mathbf{C G}$ & Sik & Arp & \\
\hline 1. & Aphelaria & 1 & - & - & - & - & - & - & - & - & - & - & - & - & 1 \\
\hline 2. & Artomyces & 1 & - & - & - & - & - & - & - & - & - & - & - & - & 1 \\
\hline 3. & Clavaria & 6 & 3 & 1 & - & - & 1 & - & 1 & - & 1 & - & - & - & 13 \\
\hline 4. & Clavariadelphus & 2 & - & - & 1 & - & - & - & - & - & - & - & - & - & 3 \\
\hline 5. & Clavulina & 8 & 2 & - & - & 1 & - & 2 & - & - & - & - & - & - & 13 \\
\hline 6. & Clavulinopsis & 7 & 4 & 1 & 1 & - & - & 2 & - & - & - & - & - & - & 15 \\
\hline 7. & Deflexula & - & - & - & - & - & - & 1 & - & - & - & - & - & - & 1 \\
\hline 8. & Gloeocantharellus & - & - & - & - & - & - & - & - & - & 1 & - & - & - & 1 \\
\hline 9. & Lachnocladium & 1 & - & - & 1 & - & - & 1 & - & - & - & - & - & - & 3 \\
\hline 10. & Lentaria & 1 & - & - & - & - & - & - & - & 1 & - & - & - & - & 2 \\
\hline 11. & Multiclavula & 1 & - & - & - & - & - & - & - & - & - & - & - & - & 1 \\
\hline 12. & Phaeoclavulina & 1 & - & - & - & 1 & 1 & - & - & - & - & - & - & - & 3 \\
\hline 13. & Pterula & - & - & - & - & - & 2 & - & - & - & - & - & - & - & 2 \\
\hline 14. & Ramaria & 19 & 11 & 2 & 4 & - & 1 & 1 & - & - & 5 & - & 3 & 5 & 51 \\
\hline 15. & Ramariopsis & 3 & 1 & - & - & 2 & 1 & 1 & - & - & - & 1 & - & - & 8 \\
\hline 16. & Scytinopogon & - & - & - & - & - & - & 1 & - & - & - & - & - & - & 1 \\
\hline 17. & Typhula & 6 & 4 & - & - & - & - & 1 & - & - & - & - & - & - & 11 \\
\hline & Total & $\mathbf{5 7}$ & $\mathbf{2 5}$ & $\mathbf{4}$ & $\mathbf{7}$ & $\mathbf{4}$ & $\mathbf{6}$ & $\mathbf{1 0}$ & $\mathbf{1}$ & $\mathbf{1}$ & $\mathbf{7}$ & $\mathbf{1}$ & $\mathbf{3}$ & $\mathbf{5}$ & $\mathbf{1 3 1}$ \\
\hline
\end{tabular}

(UK = Uttarakhand, HP= Himachal Pradesh, JK= Jammu and Kashmir, Meg =Meghalaya, MP = Madhya Pradesh, MS $=$ Maharashtra, $\mathrm{WB}=$ West Bengal, UP $=$ Uttar Pradesh, Ker $=$ Kerala, Sik =Sikkim, Arp =Arunachal Pradesh)

Occurrence and distribution of Aphelaria tuberosa and Artomyces pyxidatus are rare, only one species these fungi were reported in literature. A. tuberosa was collected on soil from Uttarakhand (Thind and Sukh Dev, 1956), while A. pyxidatus was collected on wood from the same state (Butler and Bisby, 1931). Out of 13 species of Clavaria reported, 6 are from Uttarakhand these are $C$ acuta, $C$. amoenoides, $C$. fragilis, $C$. vermicularis, $C$. zollingeri and C. indica (Corner et al., 1956; Corner et al., 1958; Thind and Sukh Dev, 1956; Thind and Anand, 1956c). Three species were reported from Himachal Pradesh including C. angulispora, C. cretacea and $C$. crosslandii (Sharma and Munjal, 1977). One species each were reported from Uttar Pradesh, West Bengal, and Jammu \& Kashmir these are, $C$. gollanii, $C$. incarnate and C. jacquemontii (Butler and Bisby, 1931; Thind and Rattan, 1967; Leveille, 1844). An unidentified species was reported on dead $\operatorname{logs}$ of Tectona grandis and Terminalia tomentosa from Kolhapur, Maharashtra (Parndekar, 1964). Clavaria versatilis =Ramaria versatilis was reported from Kerala (Mohanan, 2011).

Out 3 species of Clavariadelphus reported from India, one species, $C$. himalayensis was reported from Meghalaya (Methven, 1989) and another two, $C$. junceus and $C$. truncatus from Uttarakhand (Thind and Anand, 1956b; Thind and Sukh Dev, 1956). Eleven species Clavulina were reported from north and central India and West Bengal. Out of them 8 species namely, $C$. amethystinoides, $C$. bessonii, C. cartilaginea, C. cinerea, $C$. coralloides, C. hispidulosa, C. mussooriensis and $C$. subrugosa were reported from Uttarakhand (Corner et al., 1956, 1958; Thind and Anand, 1956b, 1956c; Thind and Raswan, 1958; Thind and Sukh Dev, 1956). C. cinerea was reported from Madhya Pradesh and 
Uttarakhand (Dehariya et al., 2010; Thind and Anand, 1956c). C. rugosa was reported from Himachal Pradesh (Sharma and Munjal, 1977) while C. ornatipes was reported from Himachal Pradesh and West Bengal (Berkeley, 1856; Thind and Rattan, 1967) and C. limosa from sal forest of West Bengal (Thind and Sharda, 1984).

Fifteen species of Clavulinopsis were reported from India out of them 8 species were reported from Uttarakhand these are $C$. alcicornis, $\quad C$. aurantiocinnabarina, $C$. corniculata, C. dichotoma, C. fusiformis, $C$. helvola, C. laeticolor and C. subtilis (Butler and Bisby, 1931; Thind and Anand, 1956b; Thind and Raswan, 1958; Thind and Sukh Dev, 1957b). One species, C. corniculata was reported from both Uttarakhand and Jammu \& Kashmir (Butler and Bisby, 1931; Thind and Anand, 1956b). Four species were reported from Himachal Pradesh namely $C$. amoena, $C$. fusiformis and $C$. semivestita (Sharma and Munjal, 1977; Thind and Rattan, 1967; Thind and Sharda, 1982). 2 species, $C$. laeticolor and $C$. sulcata were reported from West Bengal (Banerjee, 1947; Thind and Rattan, 1967; Thind and Sharda, 1986, 1987) and one species, C. laeticolor was reported from Meghalaya (Berkeley, 1852a).

Phaeoclavulina flaccida previously reported as Ramaria flaccida is distributed worldwide. It was a reported occurring on dead leaves and twigs of oak and pine (Thind and Anand, 1956a) and attached to the base of young teak tree (this article). 3 species, Phaeoclavulina zippelii $\equiv$ Clavaria zippelii, Pterula indica and $P$. verticillata were reported from Maharashtra (Senthilarasu, 2013a b).

Total 39 species of Ramaria were reported from 51 places in India and most of the species were from northern India (Uttarakhand and Himachal Pradesh). Out of them 19 were reported from Uttarakhand, these are: Ramaria brevispora, $R$. camelicolor, $R$. clarobrunnea, $R$. concolor, $R$. echinovirens, $R$. flava, $R$. flaviceps, $R$. flavobrunnescens, $R$. holorubella, $R$. kisantuensis, R. moelleriana, R. obtusissima, $R$. purpurissima, $R$. pusilla, $R$. rubrogelatinosa, $R$. sanguinea, $R$. subaurantiaca, $\quad R$. Subbotrytis, R. subgelatinosa (Corner et al., 1956, 1957, 1958; Khurana and Thind, 1979; Sharma and Jandaik, 1978; Thind and Anand 1956a; Thind and Rattan, 1967; Thind and Sukh Dev, $1957 \mathrm{a}$ b). Eleven species namely, $R$. aurea, $R$. concolor, $R$. flavoalba, $R$. flavoviridis, $R$. gracilis, $R$. holorubella, $R$. laevispora, $R$. perbrunnea, $R$. petersenii, $R$. pura and $R$. rubrogelatinosa were reported from Himachal Pradesh (Corner, 1966; Corner and Thind, 1961; Das, 2009; Sharma and Jandaik, 1978; Sharma et al., 1977; Thind and Rattan, 1967; Thind and Sharda, 1984). Five species each of Ramaria were reported from Arunachal Pradesh and Kerala. Species reported from Arunachal Pradesh include, $R$. camelicolor, $R$. flavobrunnescens, $\quad R$. rasilispora, $R$. sandaracina and $R$. suecica (Sharda and Thind (1986) while species reported from Kerala are: $R$. apiculata, $R$. eumorpha, $R$. flava, R. gracilis and R. pallida (Mohanan, 2011; Sharma and Jandaik, 1978; Thind et al., 1983). Two species, $R$. eumorpha, $R$. ochrochlora were reported from Jammu and Kashmir (Thind et al., 1983). R. aurea, R. brevispora and $R$. subalpina were reported from Sikkim, Himalaya (Das, 2009; Das et al., 2016). 4 species of Ramaria are reported from Meghalaya: $R$. formosa, R. obtusissima, $R$. stricta and $R$. synaptopoda (Berkeley, 1856; Sharda and Thind, 1986). One species each of Ramaria was reported from Maharashtra and West Bengal these are $R$. apiculata and R. sikkimia (Patil and Thite, 1977; Rattan and Khurana, 1978; Thite et al., 1976).

Ramariopsis kunzei $\equiv$ Clavaria kunzei was recorded from mixed conifers, redwood and 
hard wood forest. The species is distributed in North America, (including Hawaii and Puerto Rico) in Northern Michigan and California, Scotland, the Netherlands, Norway, Czechoslovakia, Germany, Poland and Russia. It has also been reported from China, India, Iran, the Solomon Islands and Australia. In North America, the distribution extends north to Canada, the species reported to be edible. In the present study this species was collected from Maharashtra and described (Fig. 5-8). Earlier it was reported from Uttarakhand (Thind and Sukh Dev, 1957a). Ramariopsis subtilis $\equiv$ Clavaria subtilis is distributed in U.S.A., North America, Europe and India on different substrates like, humus soil under tree, ferns and Eucalyptus. It was also recorded on wood residues on soil from Chhattisgarh. Two species of Ramariopsis, R. kunzei and $R$. tenuicula $\equiv$ Clavaria tenuicula were reported from Uttarakhand and Himachal Pradesh (Sharma and Munjal, 1977; Thind and Raswan, 1958; Thind and Sukh Dev, 1957a). $R$. asterella from West Bengal (Banerjee and Ganguly, 1945) and $R$. subtilis from Chhattisgarh (Tiwari et al., 2013) were also reported. In the present study the species is again collected and described from Jabalpur, Madhya Pradesh (Fig. 9-12). Ramariopsis biformis =Clavulinopsis biformis var. elongata was reported from Uttarakhand (Thind and Anand, 1956b).

The only species, Scytinopogon angulisporus $\equiv$ Clavaria angulispora was reported from West Bengal (Banerjee, 1947; De, 1991). 10 species of Typhula were reported from Himalayan regions including Himachal Pradesh, Uttarakhand and West Bengal (Darjeeling). These includes: T. himalayana, T. longispora, T. micans, T. ovata, $T$. phacorrhiza, T. pteridicola, T. pulgensis, T. sclerotioides, $T$. setipes and $T$. thindii (Corner et al., 1957; Khurana, 1980; Thind and Raswan, 1958; Thind and Sukh Dev,
1956, 1957a). Some clavarioid fungi are also edible, but biomasses in fruit bodies of these fungi are very less. Edible species includes: Ramaria subalpina (Das et al., 2016).

Records on 17 genera of clavarioid fungi were compiled from 13 states of India. Uttarakhand have shown maximum diversity of these fungi representing 13 genera and 57 species followed by Himachal Pradesh and West Bengal. Phaeoclavulina flaccida, Ramariopsis kunzei and $R$. subtilis were described and reported from Madhya Pradesh and Maharashtra.

Clavarioid frungi belonging to 17 genera namely, Aphelaria, Artomyces, Clavaria, Clavariadelphus, Clavulina, Clavulinopsis, Deflexula, Gloeocantharellus, Lachnocladium, Lentaria, Multiclavula, Phaeoclavulina, Pterula, Ramaria, Ramariopsis, Scytinopogon and Typhula were recorded from 131 places from 13 states of India. Genus Ramaria distributed widely and represented by collections from 49 places followed by Clavulinopsis (15), Clavaria and Clavulina (13 each) and, Typhula (11). Genera like Aphelaria, Artomyces, Deflexula, Gloeocantharellus, Multiclavula and Scytinopogon are least diverse in distribution and collected from only one place (Table 2).

\section{Acknowledgements}

The authors are thankful to Dr. G. Rajeshwar Rao, Director, Tropical Forest Research Institute, Jabalpur for providing the research facilities. The work presented was conducted under project ID No. 224/TFRI/2016/Patho1(22) funded by India Council of Forest Research \& Education (ICFRE), Dehradun.

\section{References}

Banerjee S.N. (1947). Fungous Flora of Calcutta suburbs I. Bull. Bot. Soc. Bengal 1: 37-54. 
Berkeley MJ (1856). Decades of fungi. Decades I-LXII. Indian fungi. Hooker's London, Journal of Botany and Kew Garden Miscellany 8: 174-280.

Butler EJ, Bisby GR (1931). The Fungi of India. Imperial Country Agriculture Research India. Science Monograph 1: 237p.

Corner EJH (1950). A monograph of Clavaria and allied genera. Annals of Botany Memoirs. 1:1-740

Corner EJH (1966). Species of Ramaria (Clavariaceae) without clampa. Transactions of the British mycological Society 49(1): 101-113.

Corner EJH, Thind KS, Anand GPS. (1956). The Clavariaceae of the Mussoorie Hills (India) II. Transactions of the British mycological Society 39(4): 475-484.

Corner EJH, Thind KS, Sukh Dev (1957). The Clavariaceae of the Mussoorie Hills India-VII. Transactions of the British mycological Society 40: 472-476.

Corner EJH, Thind KS, Sukh Dev (1958). The Clavariaceae of the Mussoorie Hills India-IX. Transactions of the British mycological Society 41: 203-206.

Das K (2009). Mushroom of Sikkim I: Barsey Rhododendraon Sanctuary. Sikkim State Biodiversity Board, Gangtok and Botanical Survey of India, Kolkata, 160p.

Das K, Manoj, Hembrom E, Dutta AK, Parihar A, Paloi S, Acharya K (2016). Ramaria subalpina (Gomphaceae): a new edible fungus from India. Phytotaxa 246(2): http://dx.doi.org/10.11646/phytotaxa.246. 2.5

De AB (1991). Distribution of Aphyllophorales in India- II. Amauroderma rugosum, Amylosporus campbellii and Scytinopogon angulisporus. Acta Bot. Croat. 50: 55-58.

Dehariya P, Choubey A, Wagay JA, Vyas D (2010). Wild mushrooms of Patharia forest of Sagar. Journal of Mycology and Plant Pathology 40(2): 179-186.

Dentinger BTM, McLaughlin DJ (2006). Reconstructing the Clavariaceae using nuclear large subunit rDNA sequences and a new genus segregated from Clavaria. Mycologia 98(5): 746-762.

Fries, E.M. 1821. Systema Mycologicum. 1:1520.

Giachini AJ, Castellano MA (2011). A new taxonomic classification for species in Gomphus sensu lato. Mycotaxon 115: 183-201.

Humpert AJ. et al., (2001). Molecular phylogenetics of Ramaria and related genera: evidence from nuclear large subunit and mitochondrial small subunit rDNA sequences. Mycologia 93: 465477.

Joseph AV, Manimohan P (1998). Rediscovery of two rare agaricoid basidiomycetes. Mycological Research 102(4): 476-478.

Khurana IPS (1980). The Clavariaceae of India. XIV. The genus Typhula. Mycologia 72(4): 708-727.

Léveille JH (1944). Fungi in Voyage dans L' Ind. par victor Jacquemont Pandantees annees 1829-1832. Tome 4: 179.

Methven AS (1989). Notes on Clavariadelphus. I. New species from India and China. Memoirs of the New York Botanical Garden 49: 152-157.

Mohanan C (2011). Macrofungi of Kerala. Kerala Forest Research Institute, Hand Book \# 27, Kerala, India, 597pp.

Parndekar SA (1964). A contribution to the fungi of Maharashtra. Journal University of Poona 26: 56-64.

Patil MS, Thite AN (1977). Fungal flora of Radhanagri, Kolhapur. Journal of Shivaji University 17: 149-162.

Petersen RH (1978). Notes on clavarioid fungi. XV. Reorganization of Clavaria, Clavulinopsis and Ramariopsis. Mycologia. 70(3):660-671.

Rattan SS, Khurana IPS (1978). The Clavarias of the Sikkim Himalayas. Bibliotheca Mycologica 66: 1-68.

Senthilarasu G (2013a). A check list of Ramaria of India and an interesting blue species from Western Ghats. Kavaka 41: 6-10.

Senthilarasu G (2013b). Two interesting Pterula species from Maharashtra, India. Mycosphere 4(4): 766-771. 
Sharda RM (1984). The genus Lentaria Corner in the Himalayas. Biovigyanam 10(2): 131-136.

Sharda RM, Thind KS (1986). Genus Ramaria in the Eastern Himalaya: subgenus Laeticolora - II. Proceedings of Indian Academy of Sciences, Plant Science, 96(6): 519-529.

Sharma AD, Munjal RL (1977b). Some fleshy fungi from Himachal Pradesh. Indian Journal of Mushroom 3: 18-21.

Sharma AD, Jandaik CL (1978). Genus Ramaria Holmsk. in Himachal Pradesh. Indian Journal of Mushroom 4:5-7.

Sharma AD, Jandaik CL, Munjal RL (1977). Some fleshy fungi from Himachal Pradesh. Indian Journal of Mushroom 3: 12-15.

Thite AN, Patil MS, More TN (1976). Some fleshy fungi from Maharashtra. Botanique 7 (2-3): 77-88.

Thind KS, Adlakha KL (1956). Thelephoraceae of the Mussoorie Hills-I. Indian Journal of mycological Research 2: 57-64.

Thind KS, Anand GPS (1956a). The Clavariaceae of the Mussoorie Hills-I. Journal of Indian botanical Society 35: 92-102.

Thind KS, Anand GPS (1956b). The Clavariaceae of the Mussoorie Hills-III. Journal of Indian botanical Society 35: 171-180.

Thind KS, Anand GPS (1956c). The
Clavariaceae of the Mussoorie Hills-IV. Journal of Indian botanical Society 35: 323-332.

Thind KS, Raswan GS (1958). The Clavariaceae of the Mussoorie Hills-X. Journal of Indian botanical Society 37 : 455-469.

Thind KS, Raswan GS (1960). The Clavariaceae of the Mussoorie Hills-XI. Proceedings of the $47^{\text {th }}$ Indian Science Congress ptIII, 318-319.

Thind KS, Rattan SS (1967). The Clavariaceae of India-XI. Proceedings of the Indian Academy of Science 66B: 143-156.

Thind KS, Sharda RM (1984). Three new species of clavarioid fungi from the Himalayas. Indian Phytopathology 37: 234-240

Thind KS, Sukh Dev (1956). The Clavariaceae of the Mussoorie Hills-V. Journal of Indian botanical Society 35: 512-521.

Thind KS, Sukh Dev (1957a). The Clavariaceae of the Mussoorie Hills-VI. Journal of Indian botanical Society 36: 92-103.

Thind KS, Sukh Dev (1957b). The Clavariaceae of the Mussoorie Hills-VIII. Journal of Indian botanical Society 36: 475-485.

Tiwari CK, Parihar J, Verma RK and Prakasham U (2013). Atlas of wood decaying fungi of central India. Tropical Forest Research Institute, Jabalpur, MP, $166 \mathrm{p}$.

\section{How to cite this article:}

Verma, R.K. and Vimal Pandro. 2018. Diversity and Distribution of Clavarioid Fungi in India, Three Fungi from Central India. Int.J.Curr.Microbiol.App.Sci. 7(12): 2129-2147. doi: https://doi.org/10.20546/ijcmas.2018.712.242 